\title{
Beitrag zur Kenntniss der Schwefelverbindungen des Chroms.
}

\author{
Von Max Gröger.
}

(Vorgelegt in der Sitzung am 18. März 1880.)

Die längst bekannte Thatsache, dass das Chromoxyd sich mit den Oxyden des Zinks, ${ }^{1}$ Bleis, ${ }^{2}$ Calciums, ${ }^{3}$ Bariums, ${ }^{4}$ Magnesiums, ${ }^{5}$ Cobalts ${ }^{7}$ und Nickels ${ }^{8}$ zu wohl charakterisirten Verbindungen vereinigt, in welchen dasselbe die Rolle eines Säure-Anhydrides spielt, veranlasste mich, die Frage aufzuwerfen, ob nicht das Chromsulfid in analoger Weise den Sulfiden dieser Metalle gegeniiber sich als ein Sulf-Anhydrid verhält.

Um diese Frage zu beantworten, schien es mir am einfachsten aus den besprochenen Doppelverbindungen des Chromoxyds, den sogenannten Chromiten, durch directe Einwirkung von Schwefel den Sanerstoff derselben durch Schwefel zu verdrängen. Vor Allem ist es dabei wichtig das Verhalten des freien Schwefels gegen Chromoxyd selbst, oder gegen Chromhydroxyd zu kennen; da ich ausser der Angabe von Berzelius, ${ }^{9}$ nach welcher sich durch Erhitzen eines innigen Gemenges von Schwefel und Chromhydroxyd im Vacuum Chromsulfid bildet, in derLiteratur des Chroms iiber diesen Gegenstand keine weiteren Angaben fand, dieses Verfahren mir aber unbequem erschien, so versuchte ich das Chromsulfid durch Erhitzen eines innigen Gemenges von Chrombydroxyd und Schwefel im Wasserstoffstrom darzustellen.

\footnotetext{
1 11. 2 Chancel Compt. rend. 43927.

3 Pelouze. J. B. 1851337.

4 Mitscherlich. J. f. pr. Chem. 83485.

5 Nichols. J. B. 1869894.

4 Persoz. Ann. Chim. Phys. 3725283.

7 11. ${ }^{8}$ Eiliot. Onthemagnetic combin. Göttingen 1862.

9 Berzelin: Lehrbuch 1836 Bd 3, pag. 89.
} 
Chrombydroxyd aus Chromalaunlösung mit Ammoniak gefällt wurde mit gepulvertem Schwefel innig vereinigt im Rose'schen Tiegel im Wasserstoffstrom erhitzt; nachdem aller überschüssige Schwefel verdampft war, wurde im Wasserstoffstrom erkalten gelassen, die im Tiegel gebliebene Masse, da sie sich bei der Analyse als sauerstoffhältig erwies, nochmals mit überschiussigem Schwefel innig verrieben und wieder im Wasserstoffstrom erbitzt; dann wurde diese Operation abermals wiederholt und die schliesslich erhaltene Substanz analysirt. (Ausführung der Analysen siehe unten.)

Es gaben 0.509 Substanz $0 \cdot 4165 \mathrm{Cr}_{2} \mathrm{O}_{3}$ und $1 \cdot 6425 \mathrm{BaSO}_{4}$ entsprechend

$$
\begin{array}{r}
\mathrm{Cr} \ldots 56 \cdot 15 \% \\
\mathrm{~S} \ldots \frac{44 \cdot 30}{100 \cdot 45}
\end{array}
$$

Diese Zusammensetzung entspricht aber nicht dem Chromsulfid, sondern liesse sich durch die Formel $\mathrm{Cr}_{3} \mathrm{~S}_{4}$ ausdrücken.

Dieses unerwartete Ergebniss veranlasste mich zu einer Wiederholung des Versuches mit ganz reinen Substanzen, nämlich mit aus alkalifreier Chromsäure dargestelltem Chromhydroxyd und reinem, aus Schwefelkohlenstofflösung krystalisirtem Schwefel; das nach dreimaliger Wiederholung des besprochenen Erhitzens mit Schwefel in Wasserstoffstrom erhaltene Product wurde, da, wie ich mich tiberzengte, der Wasserstoff im Rose'schen Tiegel bei grösseren Substanzmengen nicht genügend einwirken kann, in Porzellanschiffchen locker geschichtet in einem Verbrennungsofen, wie man ihn zui organischen Elementaranalyse benützt, durch 3 Stunden im Wasserstoffstrom zur Hellrothglut erhitzt; in dem, aus dem Röhrenende austretenden Wasserstoffgase liess sich durch Bleizuckerpapier Schwefelwasserstoff nachweisen.

Die resultirende Substanz wurde einer Analyse unterzogen, bei welcher

1. $0 \cdot 3946$ Substanz .... 1.2675 $\mathrm{BaSO}_{4}$

2. $0.6155 \quad \# \quad \ldots .0 .4950 \mathrm{Cr}_{2} \mathrm{O}_{3}$

ergaben, entsprechend:

\begin{tabular}{|c|c|}
\hline Cr... $55 \cdot 18 \%$ & $55 \cdot 16^{\%} \%$ \\
\hline s.... $44 \cdot 12$ & $44 \cdot 84$ \\
\hline $100 \cdot 30 \%$ & $100 \cdot 00$ \\
\hline
\end{tabular}

$$
\text { Die Formel } \mathrm{Cr}_{8} \mathrm{~S}_{4}
$$


eine Zusammensetzung, welche sich wieder sehr gut durch die Formel $\mathrm{Cr}_{3} \mathrm{~S}_{4}$ ausdrïcken lässt. Es ergibt sich nun die Frage, ob diese Formel wirlzlich einer Verbindung entspricht, welche das Endproduct der Einwirkung des Wasserstoffs auf $\mathrm{Cr}_{2} \mathrm{~S}_{3}$ ist, oder ob bei einer längeren Dauer der Einwirkung des Wasserstoffs nicht noch eine weitere Schwefelentziehung stattfindet.

Um diess zu entscheiden, wurde der Versuch ein drittes Mal mit einer neuen Menge von Chromhydroxyd, welches gelinde getrocknet worden war (da auf ein solches der Schwefel viel leichter einwirkt, als auf scharf getroknetes) in der schon besprochenen Weise wiederholt, nur wurde das Erhitzen im Wasserstoffstrome doppelt so lange, und zwardurch 6 Stunden hindurch vorgenommen. In dem austretenden Wasserstoffgase konnte am Schluss der Operation kein Schwefelwasserstoff mehr nachgewiesen werden.

Das erhaltene Product wurde analysirt, es gaben:

1. $0 \cdot 8811$ Substanz .... $0 \cdot 7148 \mathrm{Cr}_{2} \mathrm{O}_{3}$

2. $0 \cdot 7005 \quad, \quad \ldots .2 \cdot 2855 \mathrm{BaSO}_{4}$

entsprechend:

Berechnet nach $\mathrm{Cr}_{3} \mathrm{~s}_{4}$

\begin{tabular}{cc}
$\mathrm{Cr} \ldots .55 \cdot 67 \%$ & $55 \cdot 16^{\circ} \%$ \\
$\mathrm{~S} \ldots .44 \cdot 87$ & $44 \cdot 84$ \\
\hline $100 \cdot 54 \%$ & $100 \cdot 00 \%$
\end{tabular}

es findet also keine weitere Schwefelentziehung mehr statt; dadurch ist die Existenz einer Verbindung von der Formel $\mathrm{Cr}_{3} \mathrm{~S}_{4}$ in hohem Grade wahrscheinlich gemacht.

Diese Verbindung (welche man als eine Verbindung von Chromsulfür und Chromsulfid $\mathrm{Cr}_{3} \mathrm{~S}_{4}=\mathrm{CrS}$. $\mathrm{Cr}_{2} \mathrm{~S}_{3}$ betrachten, und analog dem Eisenoxyduloxyd oder Ferroferrioxyd Chromsulfürsulfid oder Chromochromisulfid nennen könnte) ist ein grauschwarzes Pulver, welches an der Luft erhitzt sich unter Erglühen und Entwicklung von Schwefeldioxyd in grünes Chromoxyd verwandelt.

$0 \cdot 6155$ Substanz hinterliessen nach dem Glühen bis zur Gewichtsconstanz $0.495 \mathrm{Cr}_{2} \mathrm{O}_{3}$, während die Theorie $\left(2 \mathrm{Cr}_{3} \mathrm{~S}_{4}+250=\right.$ $\left.8 \mathrm{SO}_{2}+3 \mathrm{Cr}_{2} \mathrm{O}_{3}\right) 0 \cdot 4944 \mathrm{Cr}_{2} \mathrm{O}_{3}$ verlangt.

Beim Schmelzen mit Natriumnitrat bildet sich Natriumehromat und Natriumsulfat.

Diese Substanz ist in Wasser unlöslich, in Salzsäure, selbst concentrirter kochender nur spurenweise löslich, in concentrirter 
Salpetersäure löst sie sich, besonders leicht beim Erwärmen ohne Schwefel abzuscheiden zur blaugrïnen, beim Erkalten violett werdenden Chromisulfat und Chrominitrat enthaltenden Flussigkeit. Auch in verdünnter Salpetersäure ist sie beim Kochen in Königswasser mit grtiner Farbe, in verdünnter Schwefelsäure (1:6) nur spurenweise löslich.

Concentrirte Schwefelsäure zersetzt beim Erwärmen die Verbindung unter Entwicklung von Schwefeldioxyd, Abscheidung von Schwefel und eines schmutzig grünlichweissen Niederschlages.

Nachdem ich so das Verhalten des Schwefels gegen Chromhydroxyd kennen gelernt hatte, ging ich daran, die Einwirkung desselben auf das Zinkchromit zu studiren.

$\mathrm{Da}$ es sehr schwer ist nach der Methode von Chance ${ }^{1}$ ein vollkommen reines, namentlich alkalifreies Präparat darzustellen, so wurde das Zinkchromit in wasserhältigem Zustande auf folgende Weise gewonnen. Lösungen von Chromsulfat und Zink. sulfat wurden in einem solchen Verhältnisse gemischt, dass auf ein Molekiul $\mathrm{Cr}_{2}\left(\mathrm{SO}_{4}\right)_{3}$ mehr als ein Molekül $\mathrm{ZnSO}_{4}$ kam, dann mit Ammoniak gefällt, der entstandene grauviolette Niederschlag abfiltrirt, gewaschen und getrocknet.

Ich überzengte mich durch vielfach modifizirte Versuche, dass es sehr schwierig ist, den Sauerstoff des Zinkchromits gänzlich durch Schwefel zu verdrängen, es gelang diess aber durch folgendes Verfahren: Obiger Niederschlag, der aus einem Gemenge von wasserhältigem Zinkchromit und Zinklhydroxyd bestand (das $\mathrm{ZnSO}_{4}$ wurde desshalb im Überflusse genommen, weil das aus dem Zinkhydroxyd bei der Einwirkung des Schwefels entstehende Zinksulfid leicht zu entfernen war, während ein Überfluss von Chromlösung respective Chromhydroxyd zur Bildung einer Schwefelungsstufe des Chroms Veranlassung gab, welche nicht von den anderen Producten zu trennen war) wurde auf das feinste zerrieben mehrmals mit Schwefel im Rose'schen Tiegel im Wasserstoffstrom in der schon beschriebenen Weise erhitzt, um ihm dadurch schon einen grossen Theil seines Sauerstoffs zu entziehen, dann wurde die Masse möglichst fein gepulvert, in Porzellanschiffchen locker geschichtet, in einer Kaliglasröhre, aus welcher

1 Compt. rend. 43.927. 
früher die Luft durch Kohlensäure ansgetrieben wurde, in darüberströmendem Schwefeldampfe durch 4 Stunden zur Hellrothglut erhitzt. Nachdem die Einwirkung voriber war, wurde der iiberschïssige Schwefel durch Erhitzen im Kohlensäurestrome abgetrieben, das Product in demselben erkalten gelassen, und dann mit concentrirter Salzsäure ausgekocht, wobei unter Schwefelwasserstoffentwicklung Zink und eine geringe Menge Chrom als Chloride in Lösung gingen; der mit Wasser ausgekochte, und bei $110^{\circ}$ getrocknete Rückstand zeigte bei der Analyse folgende Zusammensetzung: 0.8289 Grm. Substanz gaben entsprechend :

$$
0 \cdot 2266 \mathrm{ZnO}, 0 \cdot 4276 \mathrm{Cr}_{2} \mathrm{O}_{3} \text { und } 2 \cdot 5901 \mathrm{BaSO}_{4}
$$

$$
\begin{array}{cc} 
& \begin{array}{c}
\text { Berechnet nach } \\
\mathrm{ZnCr}_{2} \mathrm{~s}_{4}
\end{array} \\
\mathrm{Zn} \ldots .21 \cdot 94^{\%} \% & 21 \cdot 81^{\%} \\
\mathrm{Cr} \ldots .35 .42 & 35 \cdot 24 \\
\mathrm{~S} \ldots \ldots 42 \cdot 79 & 42 \cdot 95 \\
\hline 100 \cdot 15 & 100 \cdot 00
\end{array}
$$

Ein Haupterforderniss für das Gelingen des Versuches ist das, dass das Zinkchromit bei nicht zu hoher Temperatur getrocknet wird, so dass es noch eine leicht zerreibliche Masse bildet.

Dieser Versuch ein zweites Mal mit einer neuen Menge Zinkchromit in ganz derselben Weise wiederholt, gab ein gleiches Resultat, indem bei der Analyse für 1.101 Substanz

$$
0 \cdot 2942 \mathrm{ZnO}, 0 \cdot 5728 \mathrm{Cr}_{2} \mathrm{O}_{3} \text { und } 3 \cdot 4109 \mathrm{BaSO}_{4}
$$
erhalten wurden, entsprechend:

\begin{tabular}{cc} 
& $\begin{array}{c}\text { Berechnet nach } \\
\mathrm{ZnCr}_{2} \mathrm{~s}_{4}\end{array}$ \\
$\mathrm{Zn} \ldots \ldots 21 \cdot 44 \%$ & $21.81 \%$ \\
$\mathrm{Cr} \ldots \ldots 35 \cdot 69$ & $35 \cdot 24$ \\
$\mathrm{~S} \ldots \ldots 42 \cdot 54$ & $42 \cdot 95$ \\
\hline $99 \cdot 67$ & $100 \cdot 00$
\end{tabular}

Die Zusammensetzung dieser Substanz lässt sich daher durch die Formel $\mathrm{ZnCr}_{2} \mathrm{~S}_{4}$ ausdrücken. Dass diese Substanz eine chemische Verbindung, und niclit etwa blos ein mechanisches Gemenge von $\mathrm{ZnS}$ und $\mathrm{Cr}_{2} \mathrm{~S}_{3}$ ist, geht daraus hervor, dass ans einem solchen das Zinksulfid sich durch Salzsäure extrahiren lässt, während dieses Product in Salzsäure unlöslich, und ausserdem die violett- 
Beitrag zur Kenntniss der Schwefelverbindungen d. Chroms. 247

braune Färbung desselben eine ganz charakteristische ist. Diese Verbindung ist analog dem Zinkehromit $\left(\mathrm{ZnO}_{2} \mathrm{Cr}_{2} \mathrm{O}_{3}=\mathrm{ZnCr}_{2} \mathrm{O}_{4}\right)$ zusammengesetzt, wesshalb man dieselbe

\section{Zinksulfochromit $\left(\mathrm{ZnCr}_{2} \mathrm{~S}_{4}\right)$}

nennen könnte. Sie besitzt im pulverförmigen Zustande eine dunkel braunviolette Farbe, ist in Wasser gänzlich, in verdïnnter und concentrirter Salzsäure selbst beim Kochen fast unlöslich, in erwärmter concentrirter Salpetersäure dagegen, ebenso in Königswasser zu einer Zinksulfat und Chromisulfat enthaltenden Flüssigkeit löslich.

Beim Erhitzen an der Luft bildet sich unter Ergltihen and Entwicklung von Schwefeldioxyd grangrünes Zinkchromit 0.5394 Substanz liessen $0 \cdot 4239$ Glïhriuckstand (berechnet nach

$$
\left.\mathrm{ZnCr}_{2} \mathrm{~S}_{4}+\mathrm{O}_{12}=4 \mathrm{SO}_{2}+\mathrm{ZnCr}_{2} \mathrm{O}_{4}: 0 \cdot 4235\right) \text {. }
$$

Viel leichter war die analoge Eisenverbindung des

\section{Ferrosulfochromit $\left(\mathrm{FeCr}_{2} \mathrm{~S}_{4}\right)$}

zu erhalten. Lösungen von Chromsulfat und Ferrosulfat wurden gemischt (aus demselben Grunde, wie bei der Darstellung der Zinkverbindung wurden auf ein Molekül $\mathrm{Cr}_{2}\left(\mathrm{SO}_{4}\right)_{3}$ mehr als 1 Molekiil $\mathrm{FeSO}_{4}$ genommen) und mit Ammoniak gefällt, der entstandene Niederschlag ausgewaschen, gelinde getrocknet, auf das feinste zerrieben, und mit Schwefelpulver innig gemengt im Wasserstoffstrom erhitzt, so lange bis aller iiberfliissige Schwefel verdampft war; dann wurde die Masse neuerdings feingepnlvert, wieder mit Schwefel im Wasserstoffstrom erhitzt und dieser Process ein drittes Mal wiederholt. Die schliesslich erhaltene schwarze Masse wurde mit Salzsätre ausgekocht, so lange noch Schwefelwasserstoffentwicklung bemerkbar war; dabei ging Eisen und etwas Chrom in Lösung. Der Riickstand wurde gewaschen, bei $110^{\circ}$ getrocknet und einer Analyse unterzogen.

Bei derselben gaben

1. $0 \cdot 663$ Substanz .... $0 \cdot 1819 \mathrm{Fe}_{2} \mathrm{O}_{3}$ und $0.3557 \mathrm{Cr}_{2} \mathrm{O}_{3}$

2. $0.3262 " 1.0571 \mathrm{BaSO}_{4}$

entsprechend:

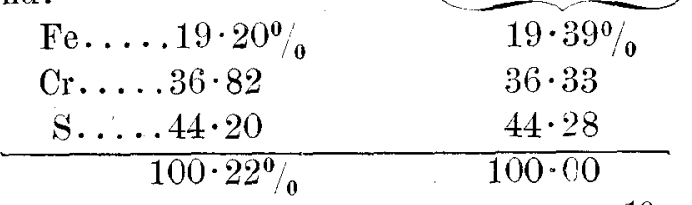


Diese Verbindung, die ein schwarzgefärbtes Pulver darstellt ist in Wasser unlöslich, in Salzsäure fast unlöslich (was wieder ein Beweis dafür ist, dass man eine chemische Verbindung, und nicht ein Gemenge von FeS und $\mathrm{Cr}_{2} \mathrm{~S}_{3}$ vor sich hat), in concentrirter Salpetersäure hingegen, so wie in Königswasser löslich. Beim Erhitzen an der Luft vergliht dieselbe unter Abgabe von Schwefeldioxyd und Hinterlassung einer braunen aus Eisenoxyd und Chromoxyd bestehenden Masse. Beim Schmelzen mit einem Gemenge von Natriumnitrat und Natriumcarbonat bildet sich Eisenoxyd, Natriumsulfat und Natriumchromat.

In ganz derselben Weise konnte auch die entsprechende Manganverbindung, das

\section{Manganosulfochromit $\left(\mathrm{MnCr}_{2} \mathrm{~S}_{4}\right)$}

dargestellt werden, indem ein Gemisch der Lösungen von Chromisulfat und Manganosulfat (letzteres wieder im Überschusse angewendet) mit Ammoniak gefällt, der entstandene Niederschlag ausgewaschen, getrocknet und mit Sehwefel im Wasserstoffstrom auf oben beschriebene Weise behandelt wurde. Das erhaltene Product wurde wieder mit Salzsäure vom tiberschisssigen Manganosulfic befreit, der unlöslich gebliebene Rüekstand getrocknet und analysirt.

Bei der Untersuchung gaben

1. 0.487 Substanz .... $0.1329 \mathrm{Mn}_{3} \mathrm{O}_{4}$ und $0.2615 \mathrm{Cr}_{2} \mathrm{O}_{3}$

2. $0.388 \quad \# \quad \ldots .1 \cdot 2433 \mathrm{BaSO}_{4}$ entsprechend:

\begin{tabular}{|c|c|}
\hline & $\begin{array}{l}\text { Berechnet a:s } \\
\mathrm{MnCH}_{2} \mathrm{~S}_{4}\end{array}$ \\
\hline $\mathrm{Mn} \ldots \ldots 19 \cdot 66^{\circ} \%$ & $19.10^{\circ} \%$ \\
\hline $\mathrm{Cr} \ldots 36 \cdot 86$ & $36 \cdot 46$ \\
\hline S.... 44.01 & $44 \cdot 44$ \\
\hline $100 \cdot 53 \%$ & $100 \cdot 00^{\circ} / 0$ \\
\hline
\end{tabular}

Diese Verbindung ist ein in Wasser und Salzsäure unlösliches, chocoladebraunes Pulver, in concentrirter Salpetersäure und Königswasser löslich. Beim Schmelzen mit Natrinmnitrat und Natriumcarbonat bildet sich Manganhyperoxyd, Natriumsulfat und Natriumchromat. Beim Erhitzen an der Luft verglimmt sie unter Entwicklung ron Schwefligsäureanhydrid, und lässt einen grün- 
grauen chromoxydhältigen Ruickstand, aus welchem sich durch Wasser Manganosulfat ausziehen lässt.

Es verhält sich also in der That das Chromsulfid $\mathrm{Cr}_{2} \mathrm{~S}_{3}$ den Monosulfiden des Zinks, Eisens und Mangan's gegeniiber, als das Sulfanhydrid derhypothetischen Sulfosäure von der Formel $\mathrm{H}_{2} \mathrm{Cr}_{2} \mathrm{~S}_{4}$.

Was die Analysen betrifft, so wurden dieselben in folgender Weise vorgenommen: Die Substanz wurde durch Schmelzen mit der sechsfachen Menge eines Gemisches aus 1. $\mathrm{NaNO}_{3}$ und 2. $\mathrm{Na}_{2} \mathrm{CO}_{3}$ im Platintiegel aufgeschlossen, die Schmelze mit Wasser ausgekocht, das eventuell ungelöst gebliebene $\mathrm{ZnO}, \mathrm{Fe}_{2} \mathrm{O}_{3}$ oder $\mathrm{MnO}_{2}$ abfiltrirt, die beiden ersteren als solche zur Wägung gebracht, letzteres in Salzsäure gelöst, als Carbonat gefällt und als $\mathrm{Mn}_{3} \mathrm{O}_{4}$ gewogen; das Filtrat wurde mit Salzsäure angesäuert, mit Alkohol versetzt, um die Chromsäure zu reduciren, das $\mathrm{Cr}$ als $\mathrm{Cr}_{2}(\mathrm{OH})_{6}$ in Porzellangefässen aus der erhitzten Fliissigkeit mit Ammoniak gefällt, das $\mathrm{Cr}_{2}(\mathrm{OH})_{6}$ abfiltrirt, gewaschen, und um es vom anhaftenden Alkali möglichst zu befreien, wieder in Salzsäure gelöst und abermals in Porzellansehalen mit Ammoniak gefällt, abfiltrirt, gewaschen, getrocknet, gegliuht und $\mathrm{Cr}_{2} \mathrm{O}_{3}$ gewogen; die beiden Filtrate vom Chromhydroxyd wurden vereinigt, mit Salzsäure angesäuert, kochend mit Chlorbarium gefăllt, das abgeschiedene $\mathrm{BaSO}_{4}$ abfiltrirt, gewaschen, getrocknet, geglïht und gewogen.

Schliesslich möchte ich mir die Bemerkung erlauben, dass ich gegriudete Ursache habe zu vermuthen, dass die Sulfide der Alkalimetalle mit Chromsulfid leicht zersetzliche Doppelverbindungen bilden, sowie auch, die Chromite des Bleies, Calciums Magnesiums etc. ein analoges Verhalten gegen Schwefel zeigen, wie das Zinkchromit, und dass ich dasselbe zum Gegenstande meines weiteren Studiums zu machen gedenke. 